\title{
Microscopic black hole detection in UHECR: the double bang signature
}

\author{
V. Cardoso ${ }^{\text {a,1 }}$, M.C. Espírito Santo ${ }^{\text {b,2 }}$, M. Paulos ${ }^{\text {b }}$, M. Pimenta ${ }^{\text {b,c,* }}$, B. Tomé ${ }^{\text {b,3 }}$ \\ ${ }^{\text {a }}$ CFC, Universidade de Coimbra, P-3004-516 Coimbra, Portugal \\ ${ }^{\mathrm{b}}$ LIP, Av. Elias Garcia 14-1, Lisboa 1000-149, Portugal \\ ${ }^{c}$ IST, Av. Rovisco Pais, Lisboa 1049-001, Portugal
}

Received 21 June 2004; received in revised form 15 September 2004; accepted 17 September 2004

Available online 30 October 2004

\begin{abstract}
According to recent conjectures on the existence of large extra dimensions in our universe, black holes could be produced during the interaction of Ultra High Energy Cosmic Rays with the atmosphere. However, and so far, the proposed signatures are based on statistical effects, not allowing identification on an event by event basis, and may lead to large uncertainties. In this note, events with a double bang topology, where the production and instantaneous decay of a microscopic black hole (first bang) is followed, at a measurable distance, by the decay of an energetic tau lepton (second bang) are proposed as an almost background free signature. The characteristics of these events and the capability of large cosmic ray experiments to detect them are discussed.
\end{abstract}

(C) 2004 Elsevier B.V. All rights reserved.

\section{Introduction}

Recent attempts to solve the hierarchy problem rely on the existence of extra dimensions in our

\footnotetext{
* Corresponding author. Address: LIP, Av. Elias Garcia 141, Lisboa 1000-149, Portugal.

E-mail address: pimenta@lip.pt (M. Pimenta).

${ }^{1}$ FCT grant SFRH/BPD/14483/2003.

${ }^{2}$ FCT grant SFRH/BPD/5577/2001.

${ }^{3}$ FCT grant SFRH/BPD/11547/2002.
}

universe, thereby lowering the fundamental Planck scale down to $\mathrm{TeV}$ energies [1]. In such scenarios, gravity should get stronger at shorter distances, and therefore new phenomena should arise in $\mathrm{TeV}$-scale experiments. One of the most interesting of these new phenomena is the production of black holes in collisions where the centre-of-mass energy is higher than $1 \mathrm{TeV}$ [2]. In this context, Ultra High Energy Cosmic Rays (UHECR) stand out naturally as the best candidates for black hole 
production, through the interaction with matter in the atmosphere [3].

The detection of events signaling black hole production, as well as their non-observation, would be of extreme importance, allowing to put constraints on the number of extra dimensions and on the effective Planck scale. However,and so far, the proposed signatures of black hole production are based on statistical effects (rates and angular distributions), not allowing identification on an event by event basis, and may lead to large uncertainties [4]. In this note events with a double bang topology are proposed as an almost background free signature. We shall elaborate upon a particular type of event, characterised by the production and instantaneous decay of a microscopic black hole (first bang) followed, at a measurable distance, by the decay of an energetic tau lepton (second bang). The characteristics of such events, and the capability of large ground-based cosmic ray experiments like Auger [5] or of the future space-based cosmic ray observatories EUSO [6] and OWL [7] to detect them are discussed below.

\section{The first bang: production and decay of microscopic black holes}

The interaction of ultra high energy cosmic neutrinos in the atmosphere has been pointed out as a good channel for the study of microscopic black hole production [8]. In the proposed scenario energetic neutrinos $\left(E_{v} \sim 10^{6}-10^{12} \mathrm{GeV}\right)$ interact deeply in the atmosphere (cross-section $\sim 10^{3}$ $10^{7} \mathrm{pb}$ ) producing microscopic black holes with a mass of the order of the neutrino-parton centreof-mass energy $(\sqrt{s} \sim 1-10 \mathrm{TeV})$. The rest lifetime of these black holes is so small $\left(\tau \sim 10^{-27} \mathrm{~s}\right)$ that an instantaneous thermal and democratic decay can be assumed. The average decay multiplicity $(\langle N\rangle)$ is a function of the parameters of the model (Planck mass $M_{\mathrm{D}}$, black hole mass $M_{\mathrm{BH}}$, number of extra dimension $n$ ) and typical values of the order of 5-20 are obtained in large regions of the parameter space. A large fraction of the decay products are hadrons $(\sim 75 \%)$ but there is a non negligible number of charged leptons $(\sim 10 \%)$
$[8,9]$. The energy spectra of such leptons in the black hole centre-of-mass reference frame peaks around $M_{\mathrm{MBH}} / N$.

The production of tau leptons in black hole decays, in particular the tau energy spectrum, was simulated using the CHARYBDIS generator [10], while the black holes themselves were produced according to phase-space and without assuming an explicit $v N \rightarrow \mathrm{BH}+X$ cross-section, as explained in Section 4. Although the CHARYBDIS generator was developed to simulate the production and decay of black holes in hadron colliders, the careful treatment of the black hole decay made it quite useful for the purpose of the present study. Some words about conventions and parameter setting in the CHARYBDIS generator are in order.

The number of extra dimensions $n$ is constrained by existing observational data to be $n>2$ [11] (for example $n=1$ is immediately excluded [1] since Newton's law holds at solar system scales). We shall take $n=3$ and $n=6$, but the conclusions apply equally well to higher dimensions. In this study, Planck mass values $M_{\mathrm{D}}=1,2$ and $3 \mathrm{TeV}$ were considered. At present, in the absence of a quantum theory of gravity, the only way to handle theoretically the black hole decay process is to consider black holes masses for which quantum gravity effects play a negligible role. This is the case for $M_{\mathrm{BH}}>M_{\min } \gg M_{\mathrm{D}}$. We shall however relax this constraint and consider that a semi-classical description is valid for $M_{\min }>M_{\mathrm{D}}$, as usually done in the literature. In this study we will consider $M_{\text {min }}=2 M_{\mathrm{D}}, 3 M_{\mathrm{D}}, 5 M_{\mathrm{D}}$ and $10 M_{\mathrm{D}}$. Although the produced black hole could have higher mass than the minimal one (depending on the impact parameter and the available centre-of-mass energy of the parton-parton collision), we work with fixed black hole masses, $M_{\mathrm{BH}}=2 M_{\mathrm{D}}, 3 M_{\mathrm{D}}, 5 M_{\mathrm{D}}$ and $10 M_{\mathrm{D}}$. This choice makes our results independent of the uncertainties on the extrapolation of the Parton Distribution Functions (PDFs) to very high energies and on the computation model for the black hole production cross-section. Furthermore, it is always possible to convolute the results, a posteriori, with a given $M_{\mathrm{BH}}$ spectrum.

When dealing with black holes, the only conserved parameters (at least in four dimensions, 
$n=0)$ are the total mass, angular momentum and electric charge. There is no lepton or baryon number conservation [12], which is sometimes stated by saying that a black hole has no hair. The CHARYBDIS generator conserves electric charge and allows lepton number violation. It does conserve baryon number, which however should not be a major drawback [10].

We note that there are several different definitions for the Planck mass $M_{\mathrm{D}}$ in the literature. Here we shall define it by

$M_{\mathrm{D}}=\left(2^{n-3} \pi^{n-1}\right)^{\frac{1}{n+2}} G_{n}^{-\frac{1}{2+n}}$,

where $G_{n}$ is Newton's constant on the $n+4$ universe.

CHAR YBDIS is interfaced with PYTHIA [13] and HERWIG [14] for standard particle hadronisation and decay. The PYTHIA option was selected in this study. For the termination of the decay, the CHARYBDIS flag KINCUT was set to false, with NBODY $=2$.

In Fig. 1(a) the multiplicity in black hole decays is shown for the scenario $\left(M_{\mathrm{D}}=1 \mathrm{TeV}\right.$, $n=3$ ), for different values of the black hole mass, $M_{\mathrm{BH}}$. It should be noted that these distributions were generated at fixed black hole masses, and should in general be convoluted with the mass spectrum of the produced black holes. While this mass spectrum is strongly peaked at the lowest possible values, the contribution from the high mass tail significantly affects the average multiplicity. This should be taken into account when comparing with the literature (e.g. Ref. [8]). Fig. $1(b)$ and (c) shows, for the same $\left(M_{\mathrm{D}}, n\right)$ scenario and several $M_{\mathrm{BH}}$ values, the number of expected tau leptons per event and the tau energy spectrum, respectively. It should be noted that both the tau leptons directly produced in the black hole evaporation and those resulting from the decay of heavy particles ( $W, Z$ bosons, top quarks) are taken into account. These relatively less energetic indirect taus may play an important role for very high primary neutrino energies. These distributions are mainly determined by the ratio $x=M_{\mathrm{BH}} / M_{\mathrm{D}}$ and have a moderate dependence on $n$, as shown by the fact that the average mul-

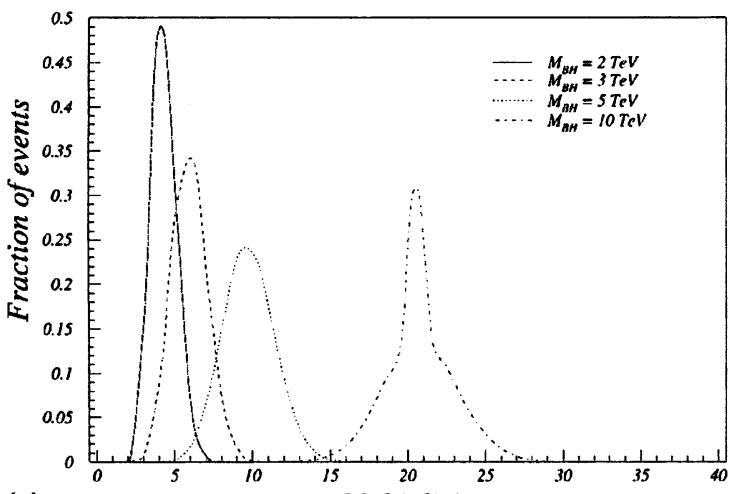

(a)
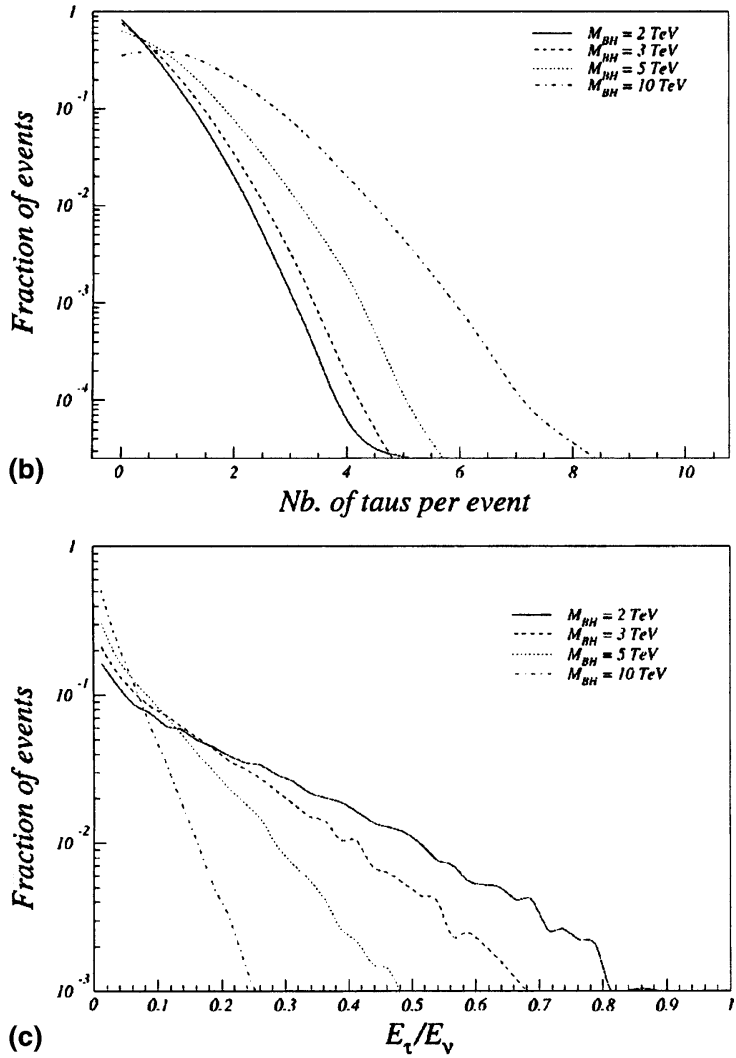

Fig. 1. For the scenario $\left(M_{\mathrm{D}}=1 \mathrm{TeV}, n=3\right)$ and different values of the black hole mass $\left(M_{\mathrm{BH}}=2,3,5,10 \mathrm{TeV}\right)$ are shown: (a) the multiplicity in black hole decays; (b) the number of taus per event $\left(\left\langle N_{\tau}\right\rangle=0.2,0.3,0.5,1.1\right.$ for $M_{\mathrm{BH}}=2,3,5,10 \mathrm{TeV}$, respectively) and (c) the fraction of the primary neutrino energy carried by taus.

tiplicity can be roughly approximated by $\langle N\rangle \sim$ $\left(\frac{M_{\mathrm{BH}}}{M_{\mathrm{D}}}\right)^{\frac{2+n}{1+n}}[2,3]$. 


\section{The second bang: the decay of energetic tau leptons}

Induced tau lepton air-showers have been proposed as a "golden" signature for cosmic neutrino detection, and thus extensively studied [15-17]. In this context, the most interesting property of energetic tau leptons is their capability of going through ordinary matter, with long decay lengths and without important energy losses. In fact, in the relevant energy range, the tau interaction length in air is much higher than its decay length, which is given by $L_{\text {decay }}=4.9 \mathrm{~km}\left(E_{\tau} / 10^{8} \mathrm{GeV}\right)$ [8]. This same property makes taus suitable for tagging microscopic black hole production in horizontal air shower events.

A detectable second bang can be produced for tau leptons with a decay length large enough for the two bangs to be well separated, but small enough for a reasonable percentage of decays to occur within the field of view. This is of course determined by the tau energy. Fig. 2(a) shows, for energies ranging from $10^{16} \mathrm{eV}$ to $10^{19} \mathrm{eV}$, the distribution of the tau decay lengths.

Another critical aspect for the detectability of the second bang is the visible energy in the tau decay. Taus can decay leptonicaly $(\sim 34 \%)$ or hadronicaly $(\sim 66 \%)$. In both cases, a fraction of the energy escapes detection due to the presence of neutrinos. In Fig. 2(b) the fraction of energy not associated to neutrinos in $\tau$ decays is shown. As observed, the average value of this energy, considering all decay modes, is of the order of $50 \%$. Hadronic decays produce a large amount of visible energy, which will be seen as an extensive air shower. For leptonic decays, not only the fraction of energy not associated to neutrinos is lower, but also only decays into electrons originate extensive air showers, leading to observable fluorescence signals.

Summarising, once a first bang is observed, the detectability of the second bang is basically determined by the tau lepton energy, which determines both the tau decay length and the energy of the second shower. The key characteristics of cosmic ray experiments are thus the field of view and the energy threshold.
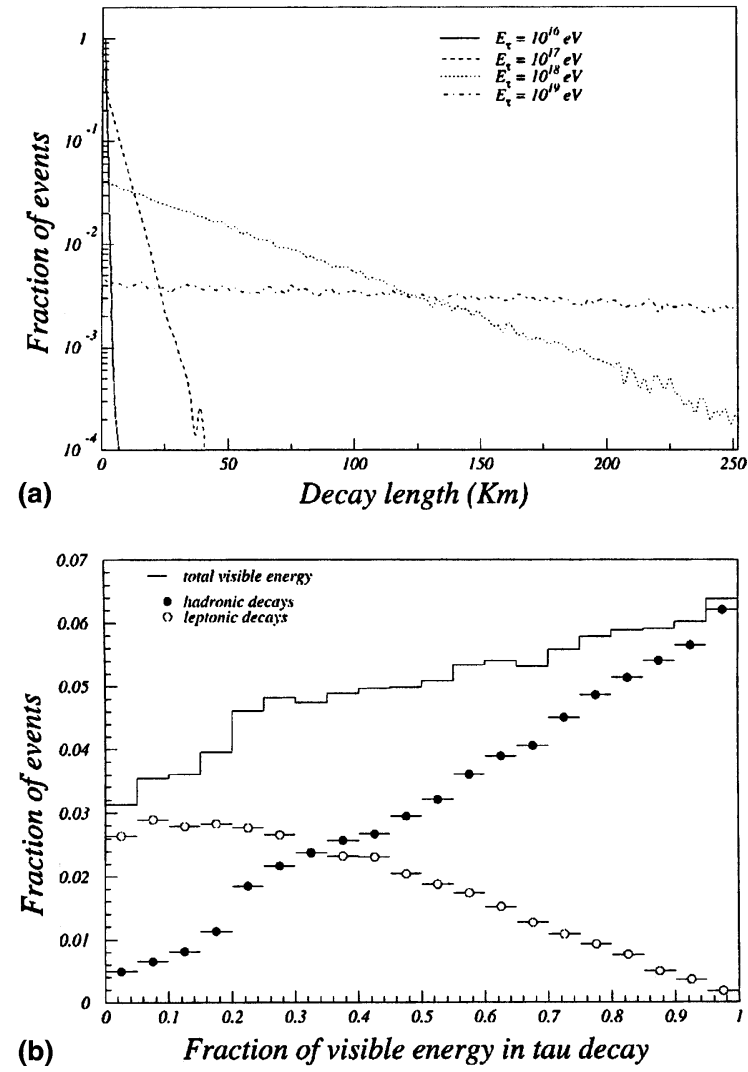

Fig. 2. Tau decay length for different energy values (a) and fraction of visible energy in $\tau$ decays (b).

\section{Observation prospects}

The observation of double bang events with two showers separated by tens or hundreds of kilometers represents an important challenge to the next generation of large scale cosmic rays experiments. The detection of the fluorescence light produced by the development of an UHECR shower in the atmosphere is a well suited technique to measure the longitudinal shower profile [18]. It has, however, two severe limitations: the very low efficiency of the energy conversion to fluorescence photons, and the atmospheric attenuation length. The first constrain restrains the application of such technique to the detection of very high energy showers in low background conditions (moonless nights). Threshold energies around $10^{19} \mathrm{eV}$ or $5 \times 10^{19} \mathrm{eV}$ 
have been quoted for Auger [5] and EUSO [6], respectively. The second constrain applies mainly to the Earth-based experiments (Auger), as the path of the fluorescence photons to space-based experiments is usually below one attenuation length. In space-based experiments, not only these attenuation effects are less critical but also a larger number of black hole induced events is typically expected, due to the larger acceptance [19]. This makes space-based experiments more promising for the detection of the double bang signature events.

In the following paragraphs EUSO will be taken as a case study, without however making any detailed simulation. In fact, double bang events were generated parameterising the shower development and the atmosphere response, in a model implemented in MATHEMATICA [20]. This approach was inspired in the method presented in the description of the SLAST simulation [21]. The GIL parameterisation [22] was used for the longitudinal shower development, while fluorescence yield follows reference [23]. Attenuation in the atmosphere was included using tables produced with LOWTRAN [24]. A cross-check with reference plots for EUSO [25] at the detector entrance was made and a reasonable agreement $(10-20 \%)$ was obtained. The results must thus be considered as an order of magnitude computation. To translate these results to OWL [7] an increase by a factor of five in expected event rates can be considered [19]. In Fig. 3, the longitudinal fluorescence profile of a double bang event at the entrance of EUSO is shown as an example.

The observation window for the double bang events is constrained by geometrical considerations (the two showers must be inside the field of view) and by the signal to noise ratio. The reconstruction of the second shower, which has an energy one order of magnitude lower than the first one, is a critical issue. However, the energy threshold for this second shower is only determined by the expected number of signal and background photons in a very restricted region of the field of view, as the second shower must be aligned with the direction of the first one.

In Fig. 4 the Confidence Level (CL) for observing the second shower is shown as a function of the

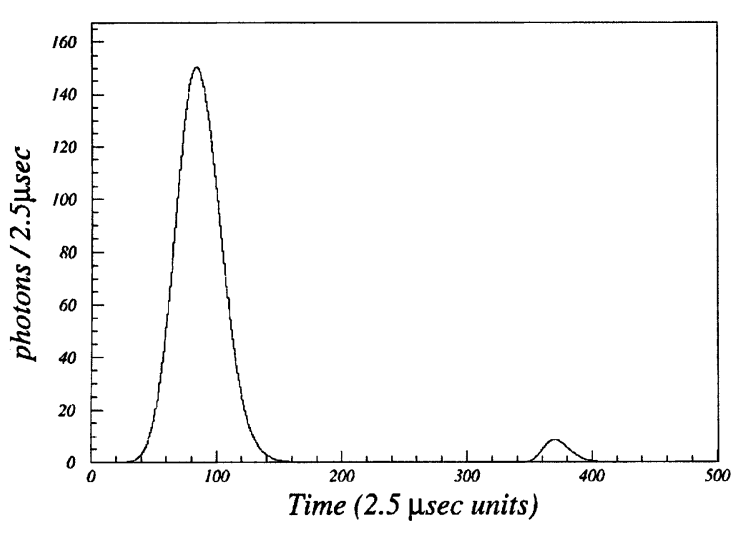

Fig. 3. Longitudinal profile of double bang event, originating from an horizontal incoming neutrino with an energy of $10^{20} \mathrm{eV}$.

shower visible energy, for horizontal showers at different altitudes. The modified frequentist likelihood ratio method [26], which takes into account not only the total number of expected signal and background events but also the shapes of the distributions, was used. Signal events were obtained using the method described above. The number of background photons has been estimated considering an expected background rate of 300-500 photons $/\left(\mathrm{m}^{2} \mathrm{~ns} \mathrm{sr}\right)$ [27] (corresponding to about $0.5-0.7$ photoelectrons per pixel in the EUSO focal surface in a time interval of $2.5 \mu \mathrm{s}$ [25]) and assuming a flat distribution. An ideal photon detection efficiency of 1.0 and a more realistic one of 0.1 were considered. Horizontal incoming neutrinos at different heights were generated, with the horizontal coordinates of the point of first interaction randomly distributed inside the EUSO field of view.

A CL of $99.7 \%(3 \sigma)$ was chosen as the criterion of visibility of the second shower. Threshold energies as low as $5 \times 10^{18} \mathrm{eV}\left(1 \times 10^{18} \mathrm{eV}\right)$ can be obtained for a photon detection efficiency of 0.1 (1.0) and a shower height of $10 \mathrm{Km}$. The height is an important parameter, as the number of detected fluorescence photons increases with the height. A factor of 20 variation in the number of detected photons can be obtained between two similar showers at low $(3 \mathrm{~km})$ and high $(20 \mathrm{~km})$ height.

To study the visibility of double bang events in EUSO, black holes produced by horizontal 

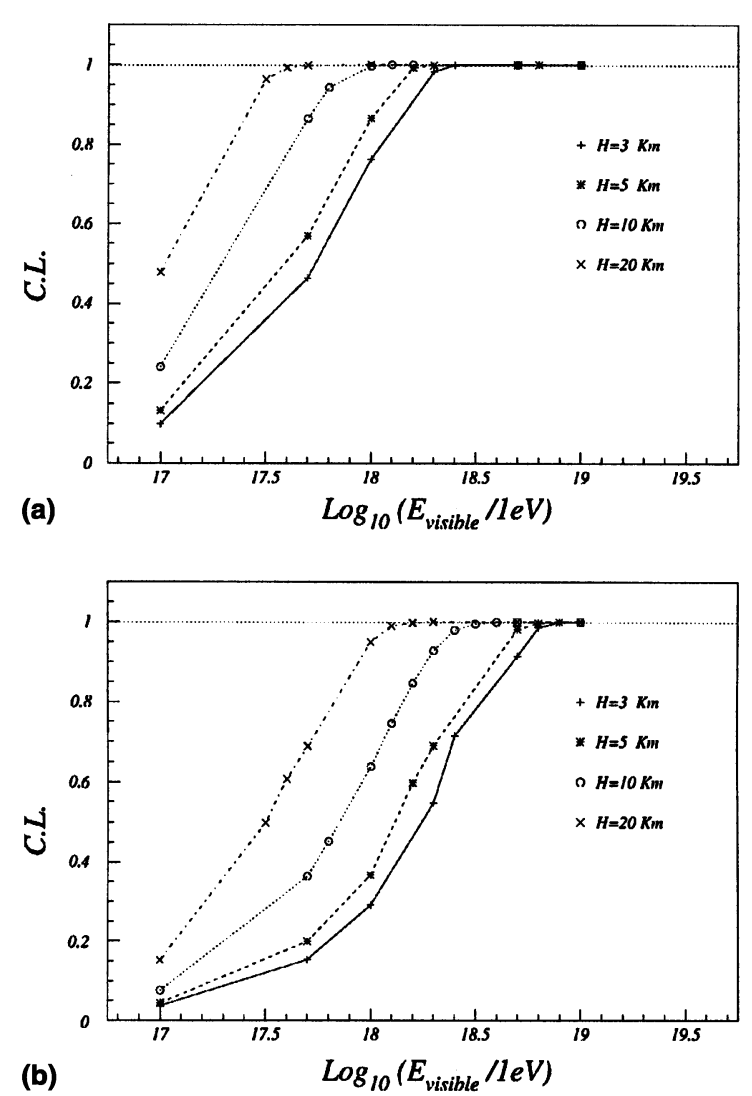

Fig. 4. Visibility confidence level of the second shower as function of its visible energy, at a simplified EUSO detector with efficiency: (a) 1.0 and (b) 0.1 , for horizontal showers at different heights. The horizontal dotted line shows the $99.7 \%$ CL $(3 \sigma)$.

incoming neutrinos were generated, $v N \rightarrow \mathrm{BH}+X$, and decayed (see Section 2). The horizontal coordinates of the point of first interaction were randomly distributed within the field of view, and the shower height was randomly chosen according to the density profile. The energy of the shower resulting from the black hole decay (first bang) was computed and tau leptons originated from this decay were followed and decayed (second bang). The visibility of the tau decay shower was established using the $\mathrm{CL}$ criterion described above. A criterion on the separation between the maxima of the two showers was further imposed, requiring the maximum of the second shower to arise on the negative slope of the first shower and more than $12.5 \mu$ s after the crossing point between the two showers.

The fraction of the black hole events with a first bang within the EUSO field of view that also have an observable second shower is shown in Fig. 5(a), as a function of the primary neutrino energy, for $\left(M_{\mathrm{D}}=1 \mathrm{TeV}, n=3, M_{\mathrm{BH}}=5 \mathrm{TeV}\right)$, and for detector efficiencies of 1.0 and 0.1 . These results take into account the fraction of events with taus in black hole decays, the tau energy spectrum and its decay length, the geometrical acceptance of EUSO and the visibility of the sec-
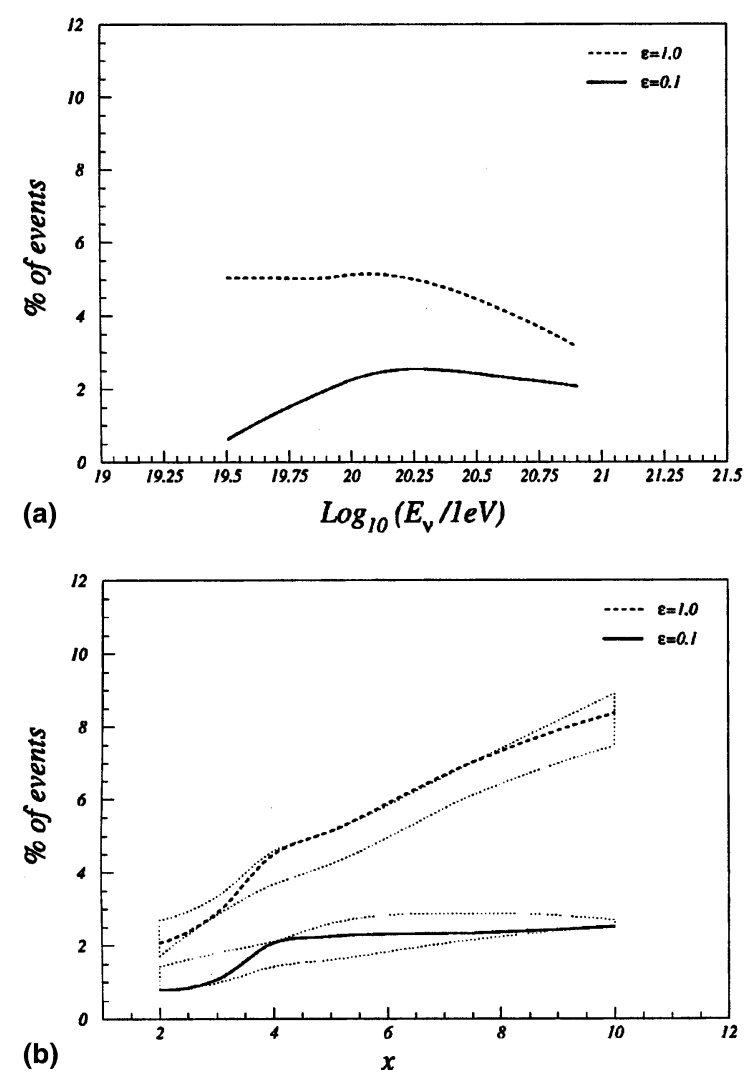

Fig. 5. Fraction of the events with a first bang within the EUSO field of view that also have a visible second bang (a) as a function of the primary neutrino energy $E_{v}$, for $\left(M_{\mathrm{D}}=1 \mathrm{TeV}\right.$, $\left.n=3, M_{\mathrm{BH}}=5 \mathrm{TeV}\right)$, and for detection efficiencies $\epsilon=0.1$ and $\epsilon=1$, (b) as a function of $x=M_{\mathrm{BH}} / M_{\mathrm{D}}$, for $E=10^{20} \mathrm{eV}$ and detection efficiencies $\epsilon=0.1$ and $\epsilon=1$. The thick lines correspond to $M_{\mathrm{D}}=1 \mathrm{TeV}, n=3$ and the dotted bands give the variation of the results when varying $M_{\mathrm{D}}$ between 1 and $2 \mathrm{TeV}$ and $n$ between 3 and 6 . 
ond shower. For a detector efficiency of 0.1 (1.0), at $E_{v}=10^{20} \mathrm{eV}$, of the order of $2 \%(5 \%)$ of the black hole induced events with a black hole decay visible in EUSO (first bang) are expected to have a visible second bang. This fraction of double bang events is limited at the lower neutrino energies by the shower visibility and at the highest by the tau lepton decay length. For relatively low energies the main limiting factor is the energy of the second bang and, in the figure, the curves corresponding to the two detector efficiencies are far apart. As the energy increases, the tau decay length increases and the second bang often escapes detection, and the curve corresponding to an efficiency of 1.0 drops considerably. On the other hand, for an efficiency of 0.1 , the favourable effect of the increase of the second bang energy is still dominant, and the two curves approach. Fig. 5(b) shows the expected fraction of double bang events as a function of $x=M_{\mathrm{BH}} / M_{\mathrm{D}}$, for $E_{v}=10^{20} \mathrm{eV}$ and two values of the detection efficiency, quantifying the dependence of the results on the values of the $\left(M_{\mathrm{D}}, n\right)$ parameters. Different values of $x$ may lead to important changes in the percentage of expected double bangs, as the black hole decay multiplicity and the tau energy spectrum are strongly dependent on $x$ (see Section 2 ). This is clearly visible in the $\epsilon=1.0$ results, where the increase in the multiplicity and, thus, in the number of taus leads to a better sensitivity for higher values of $x$. In the case $\epsilon=0.1$, this effect is compensated by the lower energies of the produced taus, and the visibility criterion described above plays an important role. The dependence on $n$ is found to be moderate.

The rate of black hole induced events depends strongly on the assumed cosmogenic and extragalactic neutrino fluxes. Values ranging from several tens to hundreds of events per year, for $M_{\mathrm{D}}=1 \mathrm{TeV}$, have been predicted for the OWL telescope [19]. In the same reference the acceptance of EUSO was estimated to be $1 / 5$ of OWL's. More specifically, taking as a reference the WaxmanBahcall bound on the incident neutrino flux, with $\mathrm{d} N_{v} / \mathrm{d} E_{v}=10^{-8} E_{v}^{-2}[28,19]$, a couple of events could be expected in EUSO, for $M_{\mathrm{D}}=1 \mathrm{TeV}$ and $n=3$ (6), for values of the ratio $x$ up to 2 (4). In the case of OWL, the sensitivity should extend, for $M_{\mathrm{D}}=1 \mathrm{TeV}$, up to $x$ of the order of 10 [19]. For $M_{\mathrm{D}}=2$, the enhancement with respect to the standard model cross-section decreases and some sensitivity could be expected only in the large $n$, very low $x$ corner. Space-based cosmic ray experiments, when compared with accelerator experiments, are less sensitive for the lowest values of the Planck mass, but give the possibility of going higher in energy, thus accessing the region $M_{\mathrm{D}}>1 \mathrm{TeV}$. Nevertheless, a good sensitivity in this region requires fluxes, cross-sections or acceptances higher than those assumed above.

Although the expected rate of double bang events due to black hole production in EUSO is rather small and would cover a relatively small region of the parameter space, the observation of just a few such events would be of the utmost importance, as almost no standard physics process has this signature. The most dangerous background comes from the $v_{\tau}$ regeneration in the Earth atmosphere $\left(v_{\tau} N \rightarrow \tau X \rightarrow v_{\tau} X\right)$ [17], which would be, in itself, a very interesting observation. However, in this type of events the second shower is expected to be more energetic than the first one. Other new physics channels may lead to similar signatures, but the observation of a reasonable number of events would give some discriminating power between different models, through the measurement of the energies of the two showers and of the distance between them.

In the case of Auger the field of view is smaller, and the observable decay lengths are restricted to about $30 \mathrm{~km}$ if the combination of two fluorescence eyes is considered. On the other hand, the lower energy threshold would allow the exploration of a lower neutrino energy window $\left(E_{v} \sim\right.$ $10^{19} \mathrm{eV}$ ) if the expected neutrino fluxes and/or the expected cross-section are higher than the values usually quoted.

\section{Conclusions}

The next generation of large cosmic ray experiments (Auger, EUSO, OWL) may have access to events with a double bang topology, an almost background free signature. This signature was explored in the framework of the production of 
microscopic black holes in the interaction of UHECR in the atmosphere. EUSO, which has an intermediate field of view between Auger and OWL, was taken as a case study.

The studies presented in this paper show that it is possible to reconstruct second bang showers above an energy threshold which is much lower than the one required to trigger on the first one. This requires that, once a very inclined shower trigger occurs, the portion of the field of view where the second bang may occur (aligned with the first shower) is also readout. In this conditions, it is shown that the second bang observation probability, once the first bang is observed, can be of the order of a few $\%$. This may lead to an expectation of a couple of events in EUSO, for $M_{\mathrm{D}}=1 \mathrm{TeV}$ and $n=3$ (6), for values of the ratio $x$ up to 2 (4). In the case of OWL, the sensitivity should extend, for $M_{\mathrm{D}}=1 \mathrm{TeV}$, up to $x$ of the order of 10 [19].

The predicted rates of double bang events are, in the case of EUSO, small and strongly dependent on the expected neutrino fluxes and on the computed cross-sections, both with large uncertainties. However, the discovery potential of such events is so high that the next generation of cosmic ray experiments should consider them in their performance studies and in the optimisation of their trigger and readout systems.

\section{References}

[1] N. Arkani-Hamed, S. Dimopoulos, G. Dvali, Phys. Lett. B 429 (1998) 263; Phys. Rev. D 59 (1999) 086004;

I. Antoniadis, N. Arkani-Hamed, S. Dimopoulos, G. Dvali, Phys. Lett. B 436 (1998) 257.

[2] P.C. Argyres, S. Dimopoulos, J. March-Russell, Phys. Lett. B 441 (1998) 96;

S. Dimopoulos, G. Landsberg, Phys. Rev. Lett. 87 (2001) 161602;

For a review see M. Cavaglia, Int. J. Mod. Phys. A 18 (2003) 1843.

[3] C. Tyler, A.V. Olinto, G. Sigl, Phys. Rev. D 63 (2001) 055001;

L.A. Anchordoqui, J.L. Feng, H. Goldberg, A. D. Shapere, hep-ph/0307228;

L.A. Anchordoqui, J.L. Feng, H. Goldberg, A.D. Shapere, Phys. Rev. D 65 (2002) 124027;

J.L. Feng, A.D. Shapere, Phys. Rev. Lett. 88 (2002) 021303 ;
A. Ringwald, H. Til, Phys. Lett. B 525 (2002) 135, hep-ph/ 0111042;

L.A. Anchordoqui, H. Goldberg, Phys. Rev. D 65 (2002) 047502;

E.-J. Ahn, M. Ave, M. Cavaglia, A. Olinto, hep-ph/ 0306008 .

[4] E.-J. Ahn, M. Cavaglia, A. Olinto, hep-ph/0312249.

[5] Auger Collaboration, The Pierre Auger Project Design Report, FERMILAB-PUB-96-024, 1996, 252. Available from: <http://www.auger.org $>$.

[6] O. Catalano, Nuov. Cimen. 24C (2) (2001) 445. Available from: http://www.euso-mission.org.

[7] J.F. Krizmanic et al.OWL/AirWatch Collaboration, Proc. 26th ICRC 2 (1999) 388. Available from: http://owl.gsfc.nasa.gov.

[8] J. Alvarez-Muñiz, J.L. Feng, F. Halzen, T. Han, D. Hooper, hep-ph/0202081.

[9] J. Tanaka, T. Yamamura, S. Asai, J. Kanzaki, Study of black holes with the ATLAS detector at the LHC, ATLPHYS-2003-037, November 2003.

[10] C.M. Harris, P. Richardson, B.R. Webber, CHARYBDIS: a black hole event generator, hep-ph/0307305; C.M. Harris, P. Kanti, J. March-Russell, in preparation.

[11] J. Hewett, M. Spiropulu, Ann. Rev. Nucl. Part Sci. 52 (2002) 397.

[12] C. Teitelboim, Phys. Rev. D 5 (1972) 2941.

[13] T. Sjostrand, P. Eden, C. Friberg, L. Lonnblad, G. Miu, S. Mrenna, E. Norrbin, Comp. Phys. Commun. 135 (2001) 238, hep-ph/0010017.

[14] G. Marchesini et al., Comp. Phys. Commun. 67 (1992) 465 ;

G. Corcella et al., J. High Energy Phys. 01 (2001) 010.

[15] D. Fargion et al., astro-ph/9704205, 1997;

D. Fargion et al., astro-ph/0305128, 2003.

[16] S. Bottai, S. Giurgula, astro-ph/0205325, 2002.

[17] H. Athar, G. Parente, E. Zas, Phys.Rev. D 62 (2000) 093010, hep-ph/0006123;

M.M. Guzzo, C.A. Moura Jr., hep-ph/0312119.

[18] A. Watson, M. Nagano, Rev. Mod. Phys. 72 (2000) 689.

[19] S.I. Dutta, M.H. Reno, I. Sarcevic, On black hole detection with the OWL/Airwatch telescope, hep-ph/0204218.

[20] Available from: <http://www.wolfram.com/products/mathematica/index.html>.

[21] D.V. Naumov, SLAST: Shower Initiated Light Attenuated to the Space Telescope, LAPP-EXP-2004-02, 2002; D. Naumov, EUSO Report SDA-REP- 015, 2003.

[22] O. Catalano et al., Proc. 27th ICRC (2001).

[23] F. Kakimoto et al., Nucl. Instrum. Methods A 372 (1996) 527.

[24] F.X. Kneizys et al., in: L.W. Abreu, G.P. Anderson (Ed.), The MODTRAN 2/3 report and LOWTRAN 7 Model, 1996.

[25] L. Scarsi et al., EUSO Collaboration, Report on the EUSO Phase A study, EUSO Report EUSO-PI-REP-002-1, 2003.

[26] A.L. Read, CERN report 2000-005, 2000, p. 81. 
[27] S. Giarrusso et al., Measurements of the UV nocturnal atmospheric background in the $300-400 \mathrm{~nm}$ wavelength band with the experiment BaBy during a transmediterranean balloon flight, Proc. 28th ICRC HE (2003) 849.
[28] E. Waxman, J.N. Bahcall, Phys. Rev. D 59 (1999) 023002, hep-ph/9807282;

E. Waxman, J.N. Bahcall, hep-ph/9902383;

K. Mannheim et al., astro-ph/9812398. 